\title{
CONSTRUCTION OF FREE SUBGROUPS IN THE GROUP OF UNITS OF MODULAR GROUP ALGEBRAS
}

\author{
Jairo Z. Gonçalves ${ }^{1}$ \\ Department of Mathematics \\ University of São Paulo \\ 66.281-Ag Cidade de S. Paulo \\ 05389-970 S. Paulo \\ São Paulo, Brazil \\ jzg@ime.usp.br
}

Donald S. Passman ${ }^{2}$

\author{
Department of Mathematics \\ University of Wisconsin-Madison \\ Van Vleck Hall \\ 480 Lincoln Drive \\ Madison, WI 53706, U.S.A \\ passman@math.wisc.edu
}

ABstract. Let $K G$ be the group algebra of a $p^{\prime}$-group $G$ over a field $K$ of characteristic $p>0$, and let $U(K G)$ be its group of units. If $K G$ contains a nontrivial bicyclic unit and if $K$ is not algebraic over its prime field, then we prove that the free product $\mathbb{Z}_{p} * \mathbb{Z}_{p} * \mathbb{Z}_{p}$ can be embedded in $U(K G)$.

\section{INTRODUCTION}

Let $K G$ be the group algebra of the group $G$ over the field $K$, and let $U(K G)$ be its group of units. Motivated by the work of Pickel and Hartley [4] , and Sehgal ([, pg. 200]) on the existence of free subgroups in the integral group ring $\mathbb{Z} G$, analogous conditions for $U(K G)$ have been intensively investigated in [ $[\underline{1},[\underline{2}]$ and [ $\underline{3}]$.

Recently Marciniak and Sehgal [ $\underline{5}$ gave a constructive method for producing free subgroups in $U(\mathbb{Z} G)$, provided $\mathbb{Z} G$ contains a nontrivial bicyclic unit. In this paper we prove an analogous result for the modular group algebra $K G$, whenever $K$ is not algebraic over its prime field $G F(p)$. Specifically, if $\mathbb{Z}_{p}$ denotes the cyclic group of order $p$, then we prove:

\footnotetext{
1. Research partially supported by CNPq - Brazil.

2. Research supported by NSF Grant DMS-9224662.

Key Words: Free groups, group algebras, group of units.

AMS Subject Classification (1991): 16S34.
} 
Theorem. Let $K$ be a field of characteristic $p>0$ containing an element $t$ transcendental over its prime subfield. Let $G$ be a group which has two elements $x, y$ such that $x$ has finite order $n, y$ does not normalize $\langle x\rangle$, and the subgroup $\left\langle x, y^{-1} x y\right\rangle$ has no $p$-torsion. If we let

$$
a=(1-x) y \widehat{x}, \quad b=\widehat{x} y^{-1}\left(1-x^{\delta}\right), \quad \widehat{x}=\sum_{i=0}^{n-1} x^{i},
$$

where $\delta=(-1)^{p}$, then $U=U(K G)$ contains

$$
\langle 1+t a, 1+t b a b, 1+t(1-b) a b a(1+b)\rangle \cong \mathbb{Z}_{p} * \mathbb{Z}_{p} * \mathbb{Z}_{p} .
$$

The assumption that $\left\langle x, y^{-1} x y\right\rangle$ has no $p$-torsion cannot be replaced by the weaker statement $\operatorname{gcd}(n, p)=1$. Indeed, let $p>2$ and let $G=P \rtimes X$ be a finite dihedral group of order $2 p$, where $P$ is cyclic of order $p$ and $X$ has order 2. Certainly $X$ is not normal in $G$ and $|X|=2$ is relatively prime to $p$. Now let char $K=p$ and let $I$ be the kernel of the natural epimorphism $K G \rightarrow K(G / P) \cong K X$. Then it is easy to see that $I^{p}=0$. Furthermore, since $K X$ is commutative, it follows that $U^{\prime} \subseteq 1+I$. But then $U^{\prime}$ is a nilpotent group of period $p$, and hence $U$ cannot contain an isomorphic copy of $\mathbb{Z}_{p} * \mathbb{Z}_{p}$. Similarly, when $p=2$, take $G=P \rtimes X \cong$ Alt $_{4}$, where $|P|=4$ and $|X|=3$.

Corollary. If $G$ is a nonabelian torsion $p^{\prime}$-group and $K$ is not algebraic over its prime subfield $G F(p)$, then $U(K G)$ contains a free noncyclic group.

\section{The Proofs}

Let $A$ be an $F$-algebra, and let $A[t]$ denote the polynomial ring over $A$ in the variable $t$. We start with:

Lemma. Assume that char $F=p>0$, and let $a$ and $b$ nonzero elements of $A$ such that $a^{2}=b^{2}=0$ and ba is not nilpotent. Let us define

$$
\begin{aligned}
& x_{1}=1+t a \\
& x_{2}=1+t b a b \\
& x_{3}=1+t(1-b) a b a(1+b) .
\end{aligned}
$$

Then, $x_{1}, x_{2}$ and $x_{3}$ are units of order $p$ in $A[t]$, and

$$
\left\langle x_{1}, x_{2}, x_{3}\right\rangle \cong \mathbb{Z}_{p} * \mathbb{Z}_{p} * \mathbb{Z}_{p} .
$$

Proof. Note that $x_{1}^{p}=x_{2}^{p}=x_{3}^{p}=1$, since $a, b a b$ and $(1-b) a b a(1+b)$ all have square zero. We now show that $x_{1}, x_{2}$ and $x_{3}$ generate $\mathbb{Z}_{p} * \mathbb{Z}_{p} * \mathbb{Z}_{p}$. 
Suppose not. Then there exists an identity of the form

$$
x_{i_{1}}^{j_{1}} x_{i_{2}}^{j_{2}} \cdots x_{i_{n}}^{j_{n}}=1
$$

with $i_{s} \in\{1,2,3\}$, with no two neighboring indices being equal, and with exponents $1 \leq j_{s} \leq p-1$. If some $i_{s}=2$, then after cyclic rotation, we may suppose that $i_{1}=2$. On the other hand, if no $i_{s}=2$, then we can conjugate equation $(*)$ by $x_{2}$ and again suppose that $i_{1}=2$. In other words, without loss of generality, we may assume that $i_{1}=2$.

An easy induction now shows that any partial product $x_{i_{1}}^{j_{1}} x_{i_{2}}^{j_{2}} \cdots x_{i_{k}}^{j_{k}}$ of the left-hand side of $(*)$ with $1 \leq k \leq n$ is a polynomial in $A[t]$ of degree $\geq 1$ with leading coefficient equal to $\pm j_{1} j_{2} \cdots j_{k} c_{k}$, where

$$
c_{k}= \begin{cases}(b a)^{r} & \text { if } i_{k}=1, \\ (b a)^{r} b & \text { if } i_{k}=2, \\ (b a)^{r}(1+b) & \text { if } i_{k}=3,\end{cases}
$$

for some $r \geq 1$. Note that all such leading coefficients are not zero since $1 \leq j_{s} \leq p-1$ and since either $c_{k}$ or $c_{k} a$ is a power of the nonnilpotent element $b a$. In particular, when $k=n$, equation $(*)$ yields a contradiction.

Proof of the Theorem. We apply the Lemma with $F=G F(p)$ and with $A=$ $G F(p) G$. Since $t \in K$ is transcendental over $F$, it follows that $K G \supseteq A[t]$. Now $a^{2}=b^{2}=0$, so it is enough to show that $b a$ is not nilpotent. We consider two cases:

(i) $p=2$. Here we have

$$
b a=\widehat{x} y^{-1}\left(1+x^{2}\right) y \widehat{x}=\widehat{x}\left(1+z^{2}\right) \widehat{x},
$$

where we set $z=y^{-1} x y$. If $b a$ is nilpotent, then by [ $\underline{6}$, Theorem 2.3.4], its trace must be zero since $\langle x, z\rangle$ is a $p^{\prime}$-group by assumption. Now the above product is the sum of the two terms $\widehat{x} \widehat{x}, \widehat{x} z^{2} \widehat{x}$, and we claim that the only contribution to the trace comes from $\widehat{x} \widehat{x}$ and is equal to $n$.

Indeed, if $x^{i} z^{2} x^{j}=1$, then we have that $z^{2}=x^{-i-j} \in\langle x\rangle$. But $z$ has odd order, so $\langle z\rangle=\left\langle z^{2}\right\rangle$, and hence $z \in\langle x\rangle$, contrary to the hypothesis. Thus the trace of $b a$ is equal to the trace of $\widehat{x}^{2}=n \widehat{x}$ and this is equal to $n$, which is not zero in $K$.

(ii) $p \neq 2$. In this case we have

$$
b a=\widehat{x} y^{-1}\left(2-x-x^{-1}\right) y \widehat{x} .
$$

As before, let us set $z=y^{-1} x y$. Then $b a=\widehat{x}\left(2-z-z^{-1}\right) \widehat{x}$, and if $b a$ is nilpotent, then by [ $\underline{6}$, Theorem 2.3.4] again, its trace must be zero since 
$\langle x, z\rangle$ is a $p^{\prime}$-group. Here the above product is the sum of the three terms corresponding to $\widehat{x} 2 \widehat{x}, \widehat{x} z \widehat{x}, \widehat{x} z^{-1} \widehat{x}$, and we claim that the only contribution to the trace of $b a$ comes from $\widehat{x} 2 \widehat{x}$, and is equal to $2 n$.

Indeed, if $x^{i} z^{\epsilon} x^{j}=1$ with $\epsilon= \pm 1$, then $z^{\epsilon}=x^{-i-j} \in\langle x\rangle$ and hence $z=y^{-1} x y \in\langle x\rangle$, contrary to the hypothesis. This proves the claim and, since $2 n$ is not zero in $K$, the result follows.

Proof of the Corollary. Suppose $U(K G)$ does not contain a noncyclic free subgroup. Then $U(K G)$ cannot contain $\mathbb{Z}_{p} * \mathbb{Z}_{p} * \mathbb{Z}_{p}$. In particular, since $G$ is not abelian, the Theorem implies that all cyclic subgroups of $G$ are normal and hence $G$ is a Hamiltonian group. Thus $p \neq 2$ and $G=A \times E \times Q_{8}$, where $A$ is an abelian group in which every element has odd order, $E$ is an elementary abelian 2-group and $Q_{8}$ is the quaternion group of order 8 . But then $K Q_{8}$ contains the direct summand $M_{2}(K)$, the full $2 \times 2$ matrix ring over $K$. Furthermore, $G L_{2}(K)$ contains nontrivial free subgroups (see for example [1]). Thus this situation cannot occur and the Corollary is proved.

\section{ACKNOWLEDGEMEnts}

The first author would like to thank Professor D. S. Passman and the Department of Mathematics of the University of Wisconsin-Madison for their hospitality and attention while this work was carried out. Also thanks to the Mathematical Sciences Department of the University of Alberta for its typing facilities which allowed this paper to be brought to final form. Finally, both authors would like to thank the referee for carefully reading the original manuscript and for rightly suggesting a number of changes and corrections.

\section{REFERENCES}

[1] Gonçalves, J. Z. Free subgroups of units in group rings, Canad. Math. Bull. 27 (1984), 309-312.

[2] Gonçalves, J. Z. Free subgroups in subnormal subgroups and the residual nilpotence of the group of units of group rings, Canad. Math. Bull. 27 (1984), 365-370.

[3] Gonçalves, J. Z. Free subgroups and the residual nilpotence of the group of units of modular and p-adic group rings, Canad. Math. Bull. 29 (1986), 321-328.

[4] Hartley, B. and Pickel, P. F. Free subgroups in the unit group of integral group rings, Canad. J. Math. 32 (1980), 1342-1352.

[5] Marciniak, Z. and Sehgal, S. K. Constructing free subgroups of integral group ring units, Proc. A.M.S., to appear.

[6] Passman, D. S. The Algebraic Structure of Group Rings, John WileyInterscience, New York, 1977.

[7] Sehgal, S. K. Topics in Group Rings, Marcel Dekker, New York, 1978. 